\title{
The decreased oxygen uptake during progressive exercise in ischemia- induced heart failure is due to reduced cardiac output rate
}

N.P.L. Rolim 3 , K.C. Mattos ${ }^{2}$ P.C. Brum², M.V.C. Baldo ${ }^{3}$, H.R. Middlekauff ${ }^{4}$ and C.E. Negrão ${ }^{1,2}$

\author{
${ }^{1}$ Instituto do Coração, Faculdade de Medicina, ${ }^{2}$ Escola de Educação Física e Esporte, \\ ${ }^{3}$ Instituto de Ciências Biomédicas, Universidade de São Paulo, São Paulo, SP, Brasil \\ ${ }^{4}$ Department of Cardiology, Los Angeles Medical School, University of California, \\ Los Angeles, CA, USA
}

Correspondence

C.E. Negrão

Unidade de Reabilitação

Cardiovascular e Fisiologia

do Exercício, InCor, FM, USP

Av. Dr. Enéas C. Aguiar, 44

05403-000 São Paulo, SP

Brasil

Fax: +55-11-3069-5043

E-mail: cndnegrao@incor.usp.br

Research supported by FAPESP (No. 01/00009-0), and in part

by Fundação Zerbini.

Received March 10, 2005 Accepted September 30, 2005

\begin{abstract}
We tested the hypothesis that the inability to increase cardiac output during exercise would explain the decreased rate of oxygen uptake $\left(\mathrm{VO}_{2}\right)$ in recent onset, ischemia-induced heart failure rats. Nine normal control rats and 6 rats with ischemic heart failure were studied. Myocardial infarction was induced by coronary ligation. $\mathrm{VO}_{2}$ was measured during a ramp protocol test on a treadmill using a metabolic mask. Cardiac output was measured with a flow probe placed around the ascending aorta. Left ventricular end-diastolic pressure was higher in ischemic heart failure rats compared with normal control rats $(17 \pm$ 0.4 vs $8 \pm 0.8 \mathrm{mmHg}, \mathrm{P}=0.0001)$. Resting cardiac index $(\mathrm{CI})$ tended to be lower in ischemic heart failure rats $(\mathrm{P}=0.07)$. Resting heart rate (HR) and stroke volume index (SVI) did not differ significantly between ischemic heart failure rats and normal control rats. Peak $\mathrm{VO}_{2}$ was lower in ischemic heart failure rats $(73.72 \pm 7.37$ vs $109.02 \pm$ $\left.27.87 \mathrm{~mL} \mathrm{~min}^{-1} \mathrm{~kg}^{-1}, \mathrm{P}=0.005\right)$. The $\mathrm{VO}_{2}$ and $\mathrm{CI}$ responses during exercise were significantly lower in ischemic heart failure rats than in normal control rats. The temporal response of SVI, but not of HR, was significantly lower in ischemic heart failure rats than in normal control rats. Peak CI, HR, and SVI were lower in ischemic heart failure rats. The reduction in $\mathrm{VO}_{2}$ response during incremental exercise in an ischemic model of heart failure is due to the decreased cardiac output response, largely caused by depressed stroke volume kinetics.
\end{abstract}

Key words - Heart failure

- Oxygen uptake rate

- Cardiac index

- Coronary ligation

- Ischemia

\section{Introduction}

Heart failure is characterized by dyspnea on exertion and exercise intolerance (1-3). In acute heart failure, such as in the setting of acute myocardial ischemia, or hypertensive crisis, dyspnea is directly related to abnormal hemodynamics. Surprisingly, in chronic heart failure, dyspnea on exertion and exercise intolerance are independent of hemodynamics and left ventricular ejection fraction at rest (4). Instead, decreased exercise ca- 
pacity in chronic heart failure has been attributed to abnormalities of the periphery, specifically the skeletal myopathy of heart failure, and abnormal muscle metabolism (5). A chain of events beginning with cardiac dysfunction and decreased cardiac output, which in turn leads to neurohumoral activation, cytokine release, and decreased muscle blood flow, all probably contribute to the skeletal myopathy of chronic heart failure. In patients with recent myocardial infarction and exercise intolerance, it is not known whether abnormal hemodynamic responses during exercise, or skeletal myopathy, or both, contribute to exercise intolerance (6). That is, it is not known at what point central mechanisms of exercise dysfunction (low cardiac output, and elevated filling pressures) give way to peripheral abnormalities.

In the present study, we tested the hypothesis that at 5 weeks after myocardial infarction, diminished cardiac output still contributes to exercise dysfunction. In previous studies of exercise and heart failure, cardiac output has not been directly evaluated nor continuously measured $(7,8)$. In the present study, we measured oxygen uptake $\left(\mathrm{VO}_{2}\right)$ and cardiac output throughout progressive exercise until exhaustion in rats with ischemia-induced heart failure, 5 weeks postmyocardial infarction. We reasoned that direct cardiac output measurements with a flow probe placed around the aortic ascending portion on a beat-to-beat basis would further elucidate the actual role of cardiac output in the decreased $\mathrm{VO}_{2}$ in heart failure.

\section{Material and Methods}

\section{Animals}

The study was performed according to the Ethics Principles in Animal Research adopted by the Brazilian College of Animal Experimentation, and was approved by the Committee on Animal Research of the Bio- medical Sciences Institute of the University of São Paulo. A total of 112 male Wistar rats were selected for the study. Seventy-eight rats (Medical School, University of São Paulo, Brazil; 330 g body weight, 5 rats/ cage) were used for the experimental protocol, but only fifteen completed the study. The other rats did not complete the study because of death during the experimental procedures and the recordings were not obtained. The rats were fed standard laboratory chow and water ad libitum in a temperaturecontrolled room $\left(22^{\circ} \mathrm{C}\right)$ with a $12: 12$-h darklight cycle.

\section{Measurements and procedures}

Induction of myocardial infarction. Myocardial infarction was induced by a coronary ligation technique described elsewhere (9). Briefly, the 3-month-old Wistar rats were anesthetized with ketamine $(50 \mathrm{mg} / \mathrm{kg}$, ip $)$ and xylazine $(10 \mathrm{mg} / \mathrm{kg}$, ip), endotracheally intubated, and mechanically ventilated with room air (respiratory rate of 60-70 breaths/ min and tidal volume of $2.5 \mathrm{~mL}$ ). Left thoracotomy was performed and the left anterior descending coronary artery was ligated.

Measurements of cardiac output. Four weeks after myocardial infarction induction, the rats were submitted to a second thoracotomy. Rats were anesthetized with ketamine $(50 \mathrm{mg} / \mathrm{kg}$, ip) and xylazine $(10 \mathrm{mg} / \mathrm{kg}$, ip), endotracheally intubated, and mechanically ventilated with room air (respiratory rate of $60-70$ breaths/min and tidal volume of $2.5 \mathrm{~mL}$ ). A transverse incision was performed on the right side of the chest wall in the second intercostal space just below the axillary area. The pectoral musculature was carefully opened by following the fiber orientation to avoid damage. An ultrasonic perivascular flow probe $(2.5 \mathrm{SL}$; Transonic Systems, Inc., Ithaca, NY, USA) was placed around the ascending aorta just above the coronary arteries and connecting wires emerged through the back of the rat (Figure 
1). Body temperature was maintained at $37^{\circ} \mathrm{C}$ by heating with an infrared lamp. After surgery, rats received an antibiotic, 20,000 U penicillin, and were returned to their cages. During the experimental session, the microprobe was connected to an ultrasonic flowmeter and then to a microcomputer for direct aortic flow velocity measurement. Cardiac output was recorded at $1000 \mathrm{~Hz}$ on a beat-tobeat basis using the AT/CODAS program (DataQ Instruments, Inc., Akron, OH, USA).

Measurement of oxygen consumption. Exercise capacity is a common method to characterize cardiac reserve and function. In addition, the ramp exercise test avoids sudden alterations in neuromuscular motor unit recruitment or metabolic changes associated with incremental protocols by employing a constant and continuous increase in external work. $\mathrm{VO}_{2}$ was measured by means of expired gas analysis during a ramp protocol of progressive exercise on a treadmill with 1$\mathrm{m} / \mathrm{min}$ increments every $40 \mathrm{~s}$ and no grade until exhaustion. In a previous study from our laboratory, the time spent by the sample gas in the mask to reach the gas analyzer was $35 \mathrm{~s}$. In addition, we observed that the steady state during submaximal workloads was achieved at about $90 \mathrm{~s}$. These findings justified the use of the ramp protocol in the present study. Therefore, a metabolic mask adapted from Russell et al. (10) was used (Figure 1). The mask fit over the face of the rat and was attached behind the ears with a latex collar. A small length of polyethylene tubing (PE-280, $3 \mathrm{~mm}$ ID) was attached to the top portion of the mask in such a way that ambient air was drawn unidirectionally into the mask from around the rat's head and exhausted through the tubing just above the rat's nose. The rats were conditioned to the metabolic mask from 6 weeks of age to the time of the experimental procedure. Gas analysis was performed using an oxygen (S-3A) I) analyzer (Ametek, Pittsburgh, PA, USA). $\mathrm{VO}_{2}$ was calculated using the measured flow through the metabolic mask, the expired fraction of effluent oxygen and the fraction of oxygen in room air.

Left ventricular pressure measurement. Rats were anesthetized with ketamine (50 $\mathrm{mg} / \mathrm{kg}$, ip) and xylazine (10 mg/kg, ip) for measurements of left ventricular end-diastolic pressure (LVEDP). A fluid-filled catheter (PE10) was inserted into the right carotid artery and advanced retrogradely into the left ventricle. LVEDP signals were recorded at $1000 \mathrm{~Hz}$ on a beat-to-beat basis using AT/CODAS (DataQ Instruments). A strain gauge transducer (Statham P23Db, Hato Rey, Puerto Rico) was used for arterial pressure measurement. The transducer signal was fed to an amplifier (GPA-4 model Z Stemtech, Inc., Wood Dale, IL, USA) and further to a 10-byte analog-to-digital converter. Only animals with LVEDP $\geq 15 \mathrm{mmHg}$ were included in the ischemic heart failure group. These values of LVEDP were associated with an infarcted area of $40 \%$ (data not shown). Contractile function was studied by directly monitoring left ventricular $\mathrm{dP} / \mathrm{dt}$.

\section{Experimental protocol}

One month after surgery for the induction of myocardial infarction, an ultrasonic perivascular flow probe was placed around the ascending aorta according to the procedure described above. Five days after surgery, one cannula was inserted into the left

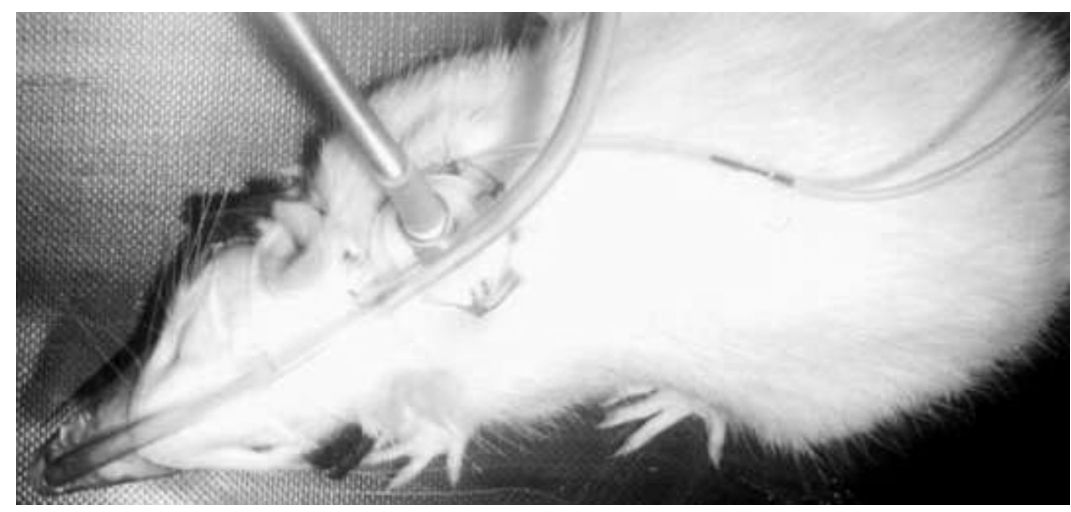

Figure 1. Rat connected to a metabolic mask and flow probe for oxygen consumption and cardiac output measurements. 
ventricle for LVEDP measurements and the study was started $24 \mathrm{~h}$ after cannula implantation. $\mathrm{VO}_{2}$ and cardiac output were then continuously evaluated during a progressive exercise test until exhaustion. Heart rate (HR) was counted from cardiac output pulses. Stroke volume was calculated from the division of cardiac output by HR, and the arteriovenous oxygen difference (a- $\mathrm{vO}_{2}$ diff.) was determined by the Fick principle.

\section{Numerical and statistical analysis}

Given the nonlinearity in the $\mathrm{VO}_{2}$ and cardiac index (CI) temporal responses during continuous measurements, we employed the Marquardt-Levenberg algorithm (implemented by means of the SigmaPlot for Windows software, version 4.01, SPSS Inc., Point Richmond, CA, USA) to fit the sigmoidal equation to the experimental data points. The physiological meaning of this curve can be determined on he basis of the four parameters that characterize the equation, where:

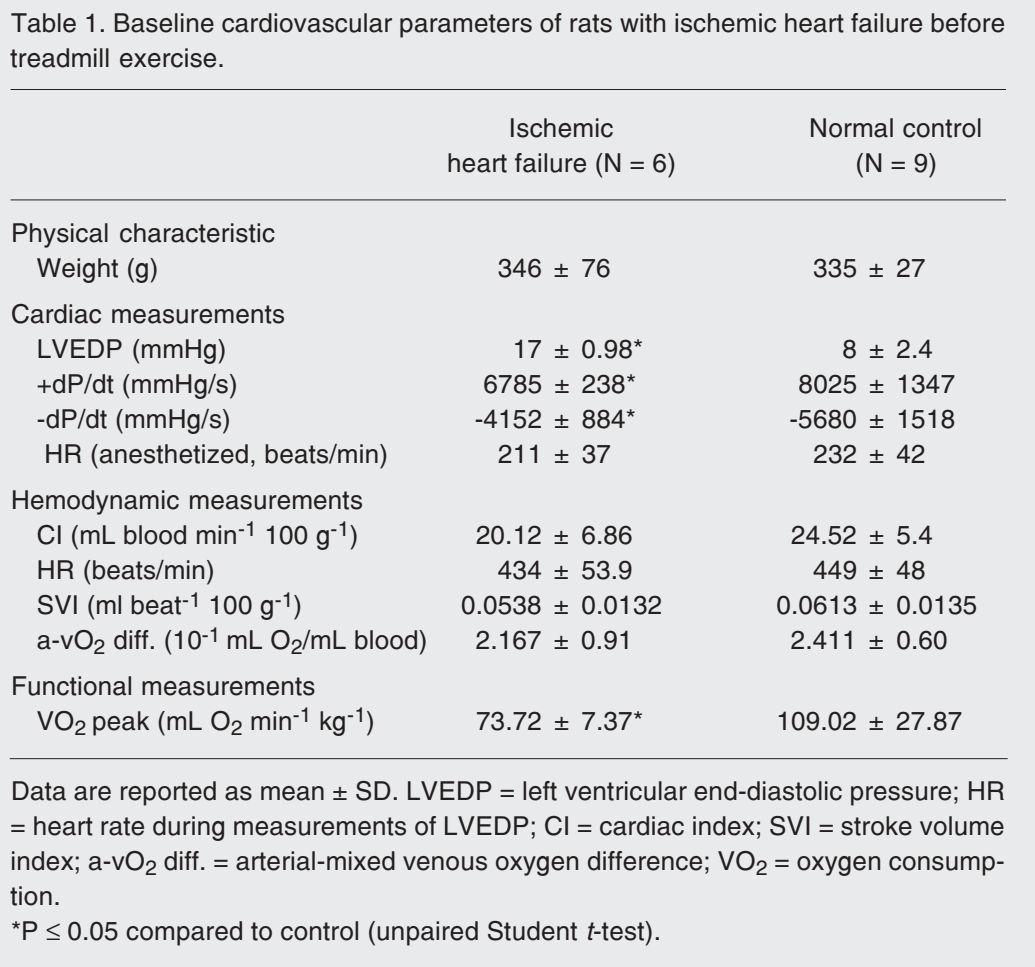

$y_{0}$ is the baseline (lower asymptote) of the variable under study (i.e., $\mathrm{VO}_{2}, \mathrm{CI}$ ), $x_{0}$ is the time the variable takes to achieve its maximal slope (latency measurement), $a$ is calculated by the difference between upper and lower asymptote ( $\Delta$ of change) and $b$ is a parameter used to calculate the curve's gain (a/4b). This sigmoidal function is given by the equation:

$$
y(x)=y_{0}+\frac{a}{1+e^{-\frac{\left(x-x_{0}\right)}{b}}}
$$

In the present study, the four parameters defining a sigmoidal curve were obtained from the best fit to each individual set of $\mathrm{VO}_{2}$ and $\mathrm{CI}$ experimental points. A mean sigmoidal curve, representing an experimental group, was obtained by averaging its parameters over the individual curves belonging to that group. Regarding the HR and stroke volume index (SVI) measures, their temporal profiles were rectilinear enough to yield a simple linear regression. For each parameter, obtained from either linear or nonlinear regression, the statistical comparison between heart failure and normal control groups was done by the unpaired Student $t$-test, with the level of significance set at $\alpha=0.05$. Data are reported as means $\pm \mathrm{SD}$.

\section{Results}

\section{Baseline measurements}

Baseline measurements of physical characteristics, left ventricular function, and functional capacity in both ischemic heart failure rats and normal control rats are summarized in Table 1. Body weight was similar in ischemic heart failure rats and normal control rats. LVEDP was significantly higher in rats with ischemic heart failure than in normal control rats. Both cardiac inotropic and lusitropic functions were decreased in rats with ischemic heart failure. Resting CI tended to be lower in ischemic heart failure rats $(\mathrm{P}=$ 
0.07). HR and SVI were not significantly different in ischemic heart failure rats and normal controls. a- $\mathrm{vO}_{2}$ diff. was similar for the two groups.

\section{Temporal responses of oxygen consumption during exercise}

The temporal responses of $\mathrm{VO}_{2}$ during progressive exercise in rats with ischemic heart failure and normal control rats are shown in Figure 2. In both groups, the $\mathrm{VO}_{2}$ response during the incremental exercise test displayed a sigmoidal pattern, which could be observed by a delayed $\mathrm{VO}_{2}$ increase at the onset of exercise paralleled by $\mathrm{VO}_{2}$ leveling off at the offset of exercise. The $\mathrm{VO}_{2}$ slope during progressive exercise was significantly lower in ischemic heart failure rats than in normal control rats $(0.29 \pm 0.15$ vs $0.66 \pm$ $0.48 \mathrm{~mL} \mathrm{O} \min ^{-1} \mathrm{~kg}^{-1} \mathrm{~s}^{-1}, \mathrm{P}=0.049$ ). In addition, the $\mathrm{VO}_{2}$ peak was significantly decreased in rats with ischemic heart failure (73.72 \pm 7.37 vs $109.02 \pm 27.87 \mathrm{~mL} \mathrm{O}_{2} \mathrm{~min}^{-1}$ $\left.\mathrm{kg}^{-1}, \mathrm{P}=0.005\right)$. Indeed, $\Delta \mathrm{VO}_{2}(a$ parameter) was significantly decreased in ischemic heart failure rats compared to normal controls $(33.97 \pm 7.64 v s 50.73 \pm 17.31 \mathrm{~mL}$ $\left.\mathrm{O}_{2} \mathrm{~min}^{-1} \mathrm{~kg}^{-1}, \mathrm{P}<0.05\right)$.

\section{Temporal hemodynamic responses during exercise}

The temporal responses of CI during exercise in ischemic heart failure rats and normal controls are shown in Figure 2. The relationship between CI and workload throughout progressive exercise also displayed a sigmoidal behavior for both groups. Similar to $\mathrm{VO}_{2}$, the increment of CI was attenuated at the onset and at the offset of exercise. The CI slope during progressive exercise was significantly lower in ischemic heart failure rats $(0.08 \pm 0.05$ vs $0.25 \pm 0.27$ $\mathrm{mL}$ blood $\left.\mathrm{min}^{-1} 100 \mathrm{~g}^{-1} \mathrm{~s}^{-1}, \mathrm{P}=0.051\right)$. In addition, peak CI was significantly lower in ischemic heart failure rats than in normal controls $(25.42 \pm 4.21$ vs $33.03 \pm 6.75 \mathrm{~mL}$ blood $\min ^{-1} 100 \mathrm{~g}^{-1}, \mathrm{P}=0.018$ ).

The temporal responses of HR and SVI are shown in Figure 3. In both groups studied, HR showed a linear regression pattern during progressive exercise. The HR slope was not significantly different in ischemic heart failure rats and normal control rats $\left(5.77 \pm 2.9\right.$ vs $5.49 \pm 3.6$ beat $\mathrm{min}^{-1} \mathrm{~s}^{-1}, \mathrm{P}=$ $0.449)$. However, peak HR was significantly lower in ischemic heart failure rats $(490 \pm$ 56.3 vs $530 \pm 23.1$ beats $/ \mathrm{min}, \mathrm{P}=0.040$ ).
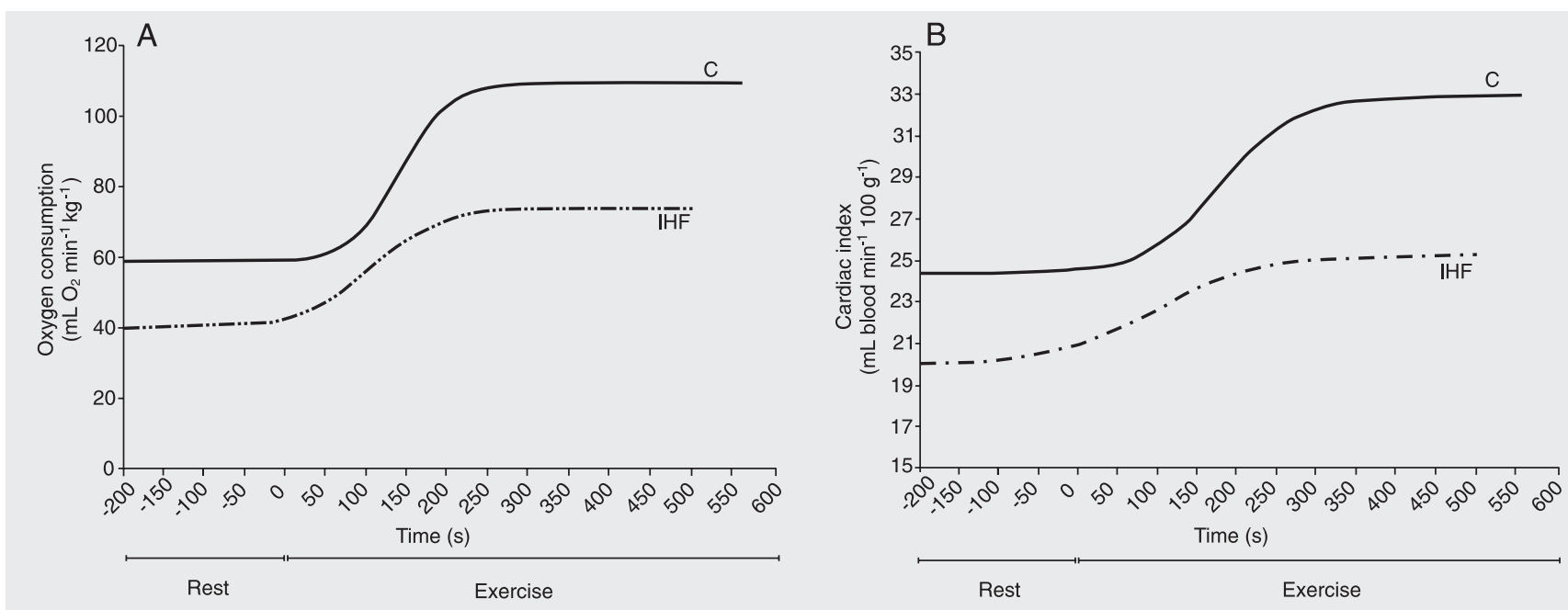

Figure 2. Time course of oxygen consumption (panel A) and cardiac output (panel B) during progressive exercise of normal control rats (C) and ischemic heart failure rats (IHF). Note that both the oxygen consumption (panel A) and cardiac output (panel B) temporal responses were reduced in ischemic heart failure rats compared to normal controls. 
Similar to HR, the SVI slope was significantly attenuated in ischemic heart failure rats $(-0.00003 \pm 0.0002$ vs $0.0004 \pm 0.0003$ $\mathrm{mL}$ beat $\left.{ }^{-1} 100 \mathrm{~g}^{-1} \mathrm{~s}^{-1}, \mathrm{P}=0.022\right)$. In addition, peak SVI was significantly lower in ischemic heart failure rats compared to normal controls $(0.0534 \pm 0.0113$ vs $0.0675 \pm 0.0153$ $\mathrm{mL}$ beat $^{-1} 100 \mathrm{~g}^{-1}, \mathrm{P}=0.038$ ).

The temporal responses of a- $\mathrm{vO}_{2}$ diff. in ischemic heart failure rats and normal control rats are shown in Figure 4. In both groups, a- $\mathrm{vO}_{2}$ diff. showed a non-linear pattern during progressive exercise. The $\mathrm{a}-\mathrm{vO}_{2}$ diff. slope did not differ between ischemic heart failure and normal control rats $(0.009 \pm$ 0.005 vs $0.034 \pm 0.048 \mathrm{~mL} \mathrm{O}_{2} \mathrm{~mL}$ blood $^{-1}$ $\left.100 \mathrm{~g}^{-1} \mathrm{~s}^{-1}, \mathrm{P}=0.117\right)$. Similarly, no significant difference was found in peak a- $\mathrm{vO}_{2}$ diff. between ischemic heart failure rats and normal controls $\left(3.12 \pm 1.13\right.$ vs $3.37 \pm 0.5710^{-1}$ $\mathrm{mL} \mathrm{O}_{2} / \mathrm{mL}$ blood, $\mathrm{P}=0.295$ ).

\section{Discussion}

In this study of the rat-infarct model of heart failure 5 weeks after the infarct, we found that the cardiac output response was blunted during exercise, and this was largely due to a fixed stroke volume. The SVI demonstrated markedly blunted kinetics during exercise, whereas HR kinetics was relatively preserved. Although abnormal cardiac output kinetics has been previously implicated in the decreased $\mathrm{VO}_{2}$ kinetics during incremental exercise $(7,8)$, we believe that the present methodological strategy provides evidence for the role of CI-mediated $\mathrm{VO}_{2}$ kinetics reduction in heart failure. First, cardiac output was directly and continuously measured in the aortic ascending portion on a beat-to-beat basis in exercising heart failure rats. Second, cardiac output and $\mathrm{VO}_{2}$ were simultaneously evaluated during progressive exercise until exhaustion.

Our methodological strategy permits a more detailed analysis of cardiac output and $\mathrm{VO}_{2}$ kinetics during progressive exercise. In contrast to previous reports, our findings demonstrate that the temporal responses of cardiac output and $\mathrm{VO}_{2}$ show a non-linear pattern rather than a linear pattern. Although nonlinearity has been previously suggested (11), our study is the first to capture it by fitting a sigmoidal curve to the experimental data points. The advantage of sigmoidal adjustment lies in the fact that additional pa-
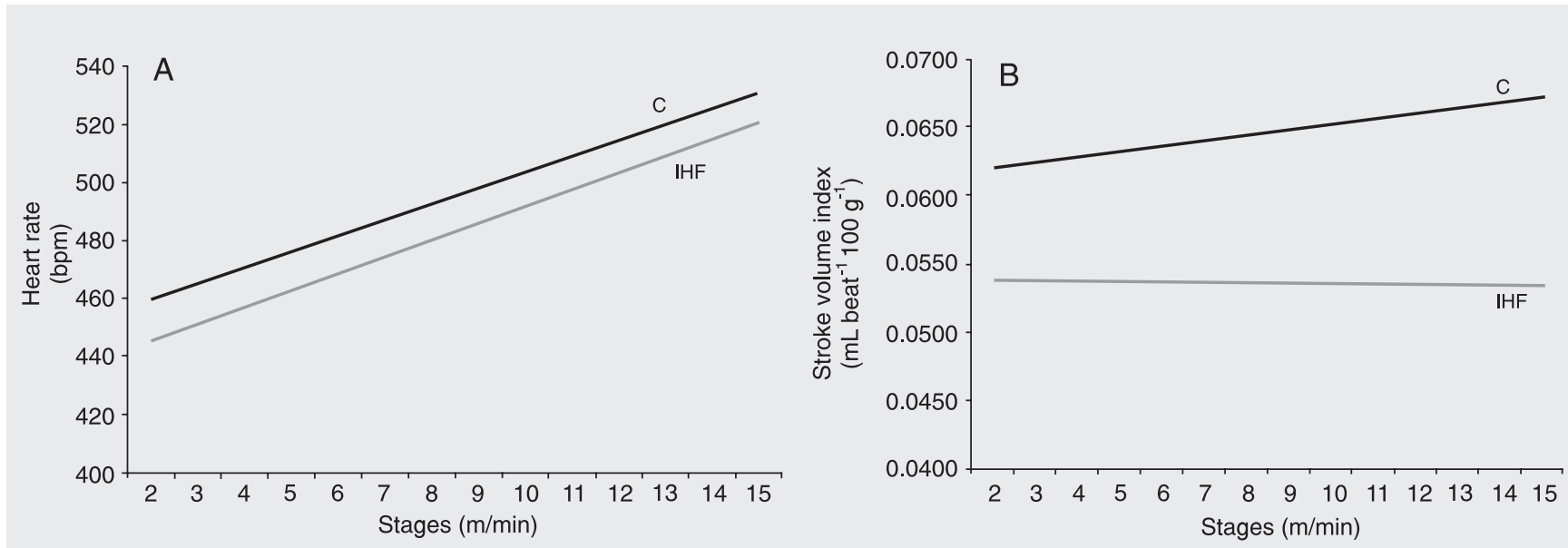

Figure 3. Time course of heart rate (HR, panel A) and stroke volume index (panel B) during progressive exercise in normal control (C) and ischemic heart failure (IHF) rats. Note that the HR responses of both groups were similar but, in contrast, stroke volume index responses were reduced in ischemic heart failure rats compared to normal controls. Panel A: C $\left(y=5.4922 x+454.05, r^{2}=0.955\right)$, IHF $\left(y=5.767 x+439.67, r^{2}=0.932\right)$. Panel B: $C\left(y=0.0004 x+0.0616, r^{2}=0.797\right), \operatorname{lHF}\left(y=-0.00003 x+0.0538, r^{2}=0.117\right)(P \leq 0.05$, unpaired Student $t$-test $)$. 
rameters such as slope, amplitude of change, and latency of the variable under study can be obtained. The sigmoidal behavior of $\mathrm{VO}_{2}$ can contribute to our understanding of certain physiological responses during exercise. For instance, the highest $\mathrm{VO}_{2}$ at submaximal exercise may indicate the time when recruitment of oxidative skeletal muscle fibers is highest. Since the cardiac response during exercise is modulated by skeletal muscle energy signaling (needed), the nonlinearity of CI could be well explained by the $\mathrm{VO}_{2}$ response. The latency at the onset of exercise may reflect the delay of oxygen consumption by the skeletal muscle. At the onset of exercise the energy supply is provided by stored ATP and creatine phosphate and the leveling off of $\mathrm{VO}_{2}$ at the peak exercise may be due to the increased recruitment of glycolytic skeletal muscle fibers.

Besides the decreased $\mathrm{VO}_{2}$ kinetics, the amplitude of change $\left(\Delta \mathrm{VO}_{2}\right.$ from rest to maximal exercise) and the maximal $\mathrm{VO}_{2}$ were reduced in exercising heart failure rats. These physiological alterations could be attributed to both central and peripheral dysfunctions. The reduced cardiac output limits the arterial oxygen transport during exercise in heart failure, which, in turn, provokes a mismatch between oxygen supplied and oxygen needed in skeletal muscle to respond to workload increments. Evidence accumulated in the last years has strongly indicated that heart failure provokes a muscle fiber shift from type I to type II, reduction in muscle mass, and blunted muscle vasodilatation, a phenomenon defined as skeletal muscle myopathy $(12,13)$. These alterations can explain, at least in part, the reduced maximal $\mathrm{VO}_{2}$ in heart failure.

The absence of a difference in a- $\mathrm{vO}_{2}$ diff. between ischemic heart failure rats and normal control rats suggests that the oxygen uptake from the blood vessels is not implicated in the reduced $\mathrm{VO}_{2}$ kinetics during exercise in rats with ischemia-induced heart failure. Tanabe et al. (8) showed that the rate

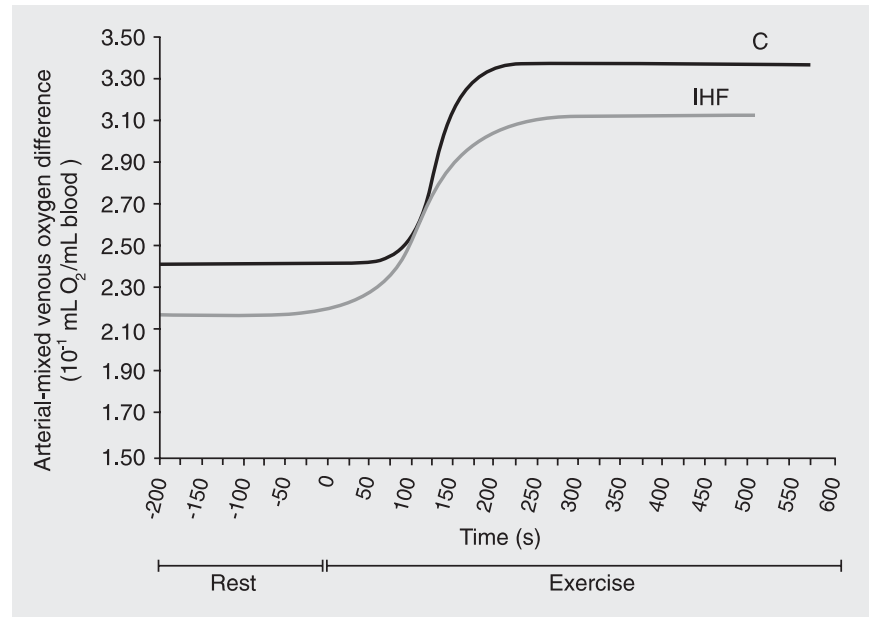

Figure 4. Time course of arterial-mixed venous oxygen difference during progressive exercise in normal control $(\mathrm{C})$ and ischemic heart failure (IHF) rats.

of $\mathrm{O}_{2}$ extraction by peripheral muscle relative to work rate increment increased with decreasing cardiac output response to exercise. But the decreased $\mathrm{O}_{2}$ supply due to severely reduced cardiac output could not be fully compensated for by the increased $\mathrm{O}_{2}$ extraction in advanced congestive heart failure (7). Although skeletal myopathy may occur within weeks following myocardial infarction (14), our findings suggest that these muscle abnormalities are not yet severe enough to play a role in the diminished oxygen consumption after recent myocardial infarction.

\section{Limitations}

We recognize several limitations of the present study. $\mathrm{a}-\mathrm{vO}_{2}$ diff. was not measured directly. However, we are confident that our $\mathrm{a}-\mathrm{vO}_{2}$ diff. findings were accurate, because $\mathrm{a}-\mathrm{vO}_{2}$ diff. was directly and continuously calculated from $\mathrm{VO}_{2}$ and cardiac output, and simultaneously measured from the mask and aortic ascending portion, respectively. The LVEDP measurements suggest that our rats were not in the advanced stage of heart failure. On the other hand, it is likely that the $\mathrm{VO}_{2}$ and $\mathrm{CI}$ kinetics was even more depressed in sicker ischemic heart failure rats. 
Although our rats were accustomed to treadmill running, we cannot guarantee that they reached the maximal exercise point during the cardiopulmonary exercise test.

\section{Perspectives}

The direct and continuous measurement of cardiovascular variables on a beat-to-beat basis, and their assessment by means of more refined mathematical methods (as illustrated in the present study), may add an important tool to the analytical apparatus currently employed to study the pathophysiology of the cardiovascular system.

The important and clinically relevant question that can be raised from the present findings is whether the decreased temporal responses of $\mathrm{VO}_{2}$ in heart failure can be reversed by a therapy that increases stroke volume and cardiac output. To accomplish this, it would be of great interest to associate pharmacological therapy that certainly improves cardiac output, such as $\beta$-blockers, angiotensin converting enzyme, $\mathrm{AT}_{1}$ blockers, with non-pharmacological therapy like aerobic exercise training to reduce skeletal myopathy in heart failure subjects. In summary, the reduction in $\mathrm{VO}_{2}$ kinetics during incremental exercise in this ischemic model of heart failure was the result of decreased cardiac output kinetics largely due to depressed stroke volume kinetics.

\section{References}

1. Colucci WS (1998). The effects of norepinephrine on myocardial biology: implications for the therapy of heart failure. Clinical Cardiology, 21: I-20-I-24.

2. Lipkin DP, Canepa-Anson R, Stephens MR et al. (1986). Factors determining symptoms in heart failure: comparison of fast and slow exercise tests. British Heart Journal, 55: 439-445.

3. Lunde PK, Sjaastad I, Schiotz Thorud HM et al. (2001). Skeletal muscle disorders in heart failure. Acta Physiologica Scandinavica, 171: 277-294.

4. Franciosa JA, Park M \& Levine TB (1981). Lack of correlation between exercise capacity and indexes of resting left ventricular performance in heart failure. American Journal of Cardiology, 47: 33-39.

5. Vescovo G (2002). Skeletal muscle response to exercise and treatment: another sibyl in the heart failure syndrome? International Journal of Cardiology, 83: 33-34.

6. Clark AL, Poole-Wilson PA \& Coats AJ (1996). Exercise limitation in chronic heart failure: central role of the periphery. Journal of the American College of Cardiology, 28: 1092-1102.

7. Koike A, Hiroe M, Adachi $\mathrm{H}$ et al. (1992). Cardiac output- $\mathrm{O}_{2}$ uptake relation during incremental exercise in patients with previous myocardial infarction. Circulation, 85: 1713-1719.

8. Tanabe Y, Nakagawa I, Ito E et al. (2002). Hemodynamic basis of the reduced oxygen uptake relative to work rate during incremental exercise in patients with chronic heart failure. International Journal of Cardiology, 83: 57-62.

9. Johns TN \& Olson BJ (1954). Experimental myocardial infarction. I. A method of coronary occlusion in small animals. Annals of Surgery, 140: 675-682.

10. Russell JC, Campagna PD \& Wenger HA (1980). Small-animal ergometer. Journal of Applied Physiology, 48: 394-398.

11. Matsumoto A, Itoh H, Yokoyama I et al. (1999). Kinetics of oxygen uptake at onset of exercise related to cardiac output, but not to arteriovenous oxygen difference in patients with chronic heart failure. American Journal of Cardiology, 83: 1573-1576.

12. Hambrecht R, Fiehn E, Yu J et al. (1997). Effects of endurance training on mitochondrial ultrastructure and fiber type distribution in skeletal muscle of patients with stable chronic heart failure. Journal of the American College of Cardiology, 29: 1067-1073.

13. Negrao CE, Rondon MU, Tinucci T et al. (2001). Abnormal neurovascular control during exercise is linked to heart failure severity. American Journal of Physiology, 280: H1286-H1292.

14. Vescovo G, Zennaro R, Sandri M et al. (1998). Apoptosis of skeletal muscle myofibers and interstitial cells in experimental heart failure. Journal of Molecular and Cellular Cardiology, 30: 2449-2459. 